\title{
Calidad física y sensorial de Coffea arábica L. variedad Colombia, perfil Nespresso AAA, en La Unión, Nariño
}

\author{
Physical and sensory quality of Coffea arabica L. variety Colombia variety, \\ Nespresso AAA profile, in the Union, Nariño
}

\section{Lady Johanna Ramos V. ${ }^{1}$; Hernando Criollo E. ${ }^{2}$}

1 Ingeniera Agrónoma, Facultad de Ciencias Agrícolas, Universidad de Nariño, Pasto, Colombia, ladyva1109@ gmail.com.

2 Profesor Asociado. Facultad de Ciencias Agrícolas, Universidad de Nariño, Pasto, Colombia, hcriolloescobar@ gmail.com.

Citar: Ramos, L.; Criollo, H. 2017. Calidad física y sensorial de Coffea arábica L. variedad Colombia, perfil Nespresso AAA, Unión Nariño. Rev. Cienc. Agr. 34(2): 83 - 97. doi: http://dx.doi. org/10.22267/rcia.173402.74.

Recibido: Noviembre 17 de $2016 . \quad$ Aceptado: Septiembre 09 de 2017.

\section{RESUMEN}

En respuesta a la necesidad de generar alternativas que permitan asegurar la calidad y diferenciación del café de Nariño, se realizó esta investigación con el objetivo de evaluar la calidad física y sensorial del café variedad Colombia, fruto rojo, sembrado a plena exposición solar por caficultores del programa Nespresso AAA 2015, en diferentes altitudes y variaciones en el beneficio, en el Municipio de la Unión, Nariño. En cada finca se cosecharon diez kilos de café maduro con color rojo intenso, se procesaron con beneficio húmedo en la misma finca, se secaron con diferentes tiempos de exposición solar y se llevaron al laboratorio de Almacafé para evaluar sus características físicas y organolépticas como: aroma, fragancia, acidez, cuerpo, sabor, sabor residual, balance, taza limpia, dulzor y puntaje del catador. Igualmente se analizaron las relaciones de los factores climáticos y agronómicos de cada finca con la calidad de taza. Toda la información se analizó mediante análisis multivariado de componentes principales (ACP) y análisis de correspondencias múltiples (ACM). Se concluyó que las condiciones de altitud, no están asociadas particularmente con los parámetros 
Ramos y Criollo. Calidad Coffea arábica L. perfil Nespresso AAA.

de calidad de la bebida del café; menores contenidos de fósforo en el suelo, menor tiempo de fermentación (16 - 17h) y secado (20 - 25h de sol), se asociaron a mayores puntajes de calidad. La compleja interacción de todas las variables asociadas a la producción del café, es la que define al final, la calidad de taza.

Palabras claves: Análisis multivariado, variables agroclimáticas, beneficio, tiempo de fermentación.

\begin{abstract}
In response to the need of generating alternatives to ensure the quality and differentiation of the Nariño coffee, this work was carried out with the objective of evaluating the physical and sensory quality of coffee variety Colombia, red fruit, sown to full sunlight by growers of Nespresso AAA program 2015, growing at different altitudes with variations in the coffee post-harvest, in the municipality of La Union, Nariño. Ten kilos of coffee fruit were harvested per farm; these fruits were processed through wet benefit and dried under different solar exposure times, and then were carried to the Almacafé laboratory to assess their physical and organoleptic characteristics such as: aroma, fragrance, acidity, body, taste, residual flavor, balance, clean cup, sweetness and score of taster. It was also analyzed the relationships between climatic and agronomic factors in each farm with the cup quality. The data were analyzed by multivariate analysis of principal components (PCA) and multiple correspondence analyses (ACM). The altitudes are not particularly associated with the quality parameters of the coffee drink; lower contents of P, less time of fermentation (16 - 17h) and drying (20 - 25h of sun), were associated with higher scores of quality. The complex interaction of all the variables associated to the coffee production of, is what defines, the cup quality.
\end{abstract}

Keywords: Multivariate analysis, soil and climatic variables, coffee processing, fermentation time.

\section{INTRODUCCIÓN}

El cultivo de café representa una de las actividades agrícolas más sobresaliente en la región suroeste de Colombia. Nariño es reconocido internacionalmente por su café de alta calidad en aroma y sabor que le imprimen a este características organolépticas únicas (Rebolledo y Vento, 2004., Oberthur et al, 2011 y Delgado et al., 2008).

De acuerdo con el Informe del Comité Departamental de Cafeteros de Nariño (2008), la economía basada en el café es fundamental para el departamento, por cuanto genera alrededor de 30 mil empleos permanentes y más de 1,2 millones de jornales al año en las épocas de recolección; Nariño cuenta con 31.940 caficultores que desarrollan su actividad en 36 municipios. El $40 \%$ del suelo de la zona cafetera nariñense se encuentra cultivado, la mayor parte de los cultivos se encuentran en pequeños predios o minifundios localizados en alturas superiores a 1.700 metros sobre el nivel del mar. En estos lugares, estas pequeñas y medianas fincas son la fuente principal de ingresos de los agricultores.

Las condiciones agroecológicas donde se ubica la caficultura, unida a las variedades cultivadas y al sistema de beneficio utilizado, permiten obtener cafés de alta calidad para atender el nicho de mercado de cafés especiales y certificados en los mercados nacionales e internacionales (Sepúlveda et al., 2013, Raynolds et al., 2007 y Raynolds, 2009). 
La altitud puede estar influenciando significativamente la composición bioquímica, la calidad física y organoléptica de la taza de café (Lara y Vaast, 2007); sin embargo, no todos los trabajos son concluyentes en el análisis de la relación entre la calidad taza y las condiciones agroecológicas del cultivo del café (Buenaventura y Castaño, 2002). El proceso de beneficio también es uno de los factores determinantes en obtención de la calidad del grano, ya que fallas en el proceso pueden originar hasta el $80 \%$ de los problemas de calidad (Aristizabal y Duque, 2006). Así mismo, un buen beneficio influye favorablemente en la obtención de un café suave, determinando la calidad de la bebida (Puerta, 2000).

El café de Nariño con las especificaciones exigidas por Nespresso AAA de Nestlé, debe poseer un perfil de aroma y sabor único y complejo para producir los cafés Grand Crus, recolección manual, fermentados y secados al sol; así ha logrado posicionarse como uno de los mejores cafés del país y garantiza la calidad del café suministrado; este café es comercializado por Almacafé y puesto a disposición del Fondo Nacional del café para su exportación. La calidad física y sensorial del café producido en Nariño, pueda atender la demanda de nichos de mercado exclusivos (Puerta, 1999; Salazar, 2000).

Los ingresos de los caficultores presentan alta vulnerabilidad a la variación de factores relacionados con la oferta ambiental (brillo solar, humedad relativa, temperatura) de las diferentes altitudes del municipio, al presentarse una relación directamente proporcional entre la altitud y el manejo en los procesos post-cosecha (beneficio) con el perfil sensorial (Donnet et al., 2007; Puerta, 2000), procesos de cosecha y post-cosecha deben realizarse bien para preservar la calidad del grano
(Rebolledo y Vento, 2004). De igual manera, Aristizabal y Duque (2006) establecieron que el beneficio es determinante en la composición química de la bebida y en el precio. El cultivador de café, además de pretender obtener una alta producción, también busca una buena calidad de la misma.

Según Puerta (1998) la calidad del café pergamino seco producido en las fincas de Colombia está influenciada por la variedad sembrada, las condiciones climáticas, el manejo agronómico y fitosanitario del cultivo, además de los procesos de cosecha y post-cosecha realizados por los caficultores.

En este sentido cada una de las características anteriormente mencionadas hacen parte de un ambiente, que rodea el sistema cafetero, el cual está en constante interacción. Aunque el ambiente puede ser un recurso para el sistema de cultivo, también puede ser una amenaza, ya que puede modificarse para favorecer o deteriorar las características sensoriales de la bebida (Arcila et al., 2007).

Todas las herramientas y alternativas que permitan mejorar los procesos y asegurar la calidad y diferenciación del café de Colombia, son herramientas importantes para mantener la competitividad y preservar la calidad física y organoléptica que han hecho del café Colombiano uno de los más apetecidos en el mercado mundial (Aristizabal y Duque, 2006).

El proceso de beneficio también es uno de los factores determinantes en obtención de la calidad del grano, ya que fallas en el proceso pueden originar hasta el $80 \%$ de los problemas de calidad (Aristizabal y Duque, 2006). Así mismo, un buen beneficio influye favorablemente en la obtención de un café suave, determinando la calidad de la bebida (Puerta, 2000). 
Las cualidades sensoriales del café más importantes son su aroma, acidez, cuerpo y sabor (Puerta 1996).

En este sentido, la presente investigación tuvo como objetivo establecer la relación entre la oferta ambiental, con las características físicas y atributos en la bebida, del café Nespresso AAA, variedad Colombia, producido en el municipio de la Unión Nariño

\section{MATERIALES Y MÉTODOS}

Localización. El presente trabajo se realizó en el Municipio de La Unión, Nororiente del Departamento de Nariño ubicado en las coor-

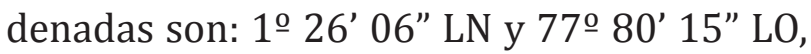
meridiano de Greenwich cuya cabecera se encuentra a una altura de $1.650 \mathrm{msnm}$ y una temperatura promedio de $19^{\circ} \mathrm{C}$.

El trabajo, se realizó con base en un estudio de caso con nueve caficultores con perfil Nespresso AAA 2015, programa de cafés especiales de calidad, los cuales pertenecen a las veredas de La Playa (3), el Sauce (3) y Buenos Aires (3).

Selección de las fincas. Del Cluster Nespresso AAA con perfil del año 2015 se seleccionaron nueve caficultores de veredas cercanas a las tres estaciones meteorológicas ubicadas en diferentes altitudes, zona baja $(<1500$ $\mathrm{msnm}$ ) en la vereda de La Playa, zona media (1501 - $1800 \mathrm{msnm}$ ) en la vereda del Sauce A y la zona alta ( $>1801 \mathrm{msnm}$ ) en la vereda de Buenos Aires, en el Municipio de la Unión, departamento de Nariño. La selección se hizo teniendo en cuenta el manejo del lote y el proceso de beneficio húmedo, variedad cultivada Colombia, fertilización química tradicional, terreno plano, cultivos a plena exposición y estado de madurez del fruto.
Información meteorológica. Se utilizó la información obtenida en las estaciones meteorológicas del Grupo de Investigaciones en Producción de Frutales Andinos, localizadas en áreas próximas a las fincas seleccionadas.

Información recopilada de las fincas. Se determinaron las características de las fincas incluyendo nombre de la finca, propietario, área total del lote, área del cultivo, altitud, coordenadas, tipo de cultivo (nueva siembra, renovación por zoca, renovación por siembra), distancias de siembra, edad del cultivo, densidad de siembra, fertilización, presencia de plagas y enfermedades, temperatura ambiente, horas de fermentación y horas de secado.

Toma de muestras. En el momento de la cosecha principal que se realiza entre abril y junio, de cada lote se cosecharon manualmente diez kilos de café maduro, los cuales se sometieron al proceso de beneficio, en cada una de las fincas.

Beneficio de las muestras. Los frutos maduros, fueron procesados por el método de beneficio húmedo, se despulparon, se pesó el café con mucilago y se midió el pH del mucilago del café.

En cada finca, el café cereza recién cosechado se despulpó y se colocó a fermentar en canecas cilíndricas de plástico previamente lavadas con agua potable; el proceso de fermentación se desarrolló en ambiente externo, en sistemas estáticos y abiertos, utilizando el método del Fermaestro de Cenicafé con el fin de registrar las horas de fermentación de cada finca. El café se lavó cuidadosamente, se pesó y se secó al sol hasta obtener una humedad final entre el 10 - 12\% usando el método Gravimet desarrollado por Cenicafé. 
En cada una de las fincas, se seleccionaron cuatro muestras de $500 \mathrm{~g}$ café pergamino seco, procesadas según las normas de Nespresso y se enviaron en empaque plástico a Almacafé para su análisis físico y sensorial.

Variables evaluadas para determinar la calidad del café. Las variables relacionadas con el análisis físico y sensorial (proceso de catación) se determinaron según las normas y procedimientos seguidas por Almacafé en la ciudad de Pasto Nariño (protocolo de catación). El perfil Nespresso AAA, se realizó en el laboratorio de Almacafé que tiene los estándares manejados por la empresa.

Análisis estadísticos. Se aplicó análisis multivariado según el método de Análisis de Componentes Principales (ACP), considerando las variables cuantitativas relacionadas con el clima y con el cultivo. Las variables relacionadas con el beneficio del grano y el análisis físico y sensorial de las muestras de café pergamino seco, se categorizaron y analizaron con el método de Análisis de Correspondencias Múltiples (ACM). Se utilizó el método de agrupamiento con base en las distancias de Ward, utilizando el software SPAD versión 5.6.

\section{RESULTADOS Y DISCUSIÓN}

Variables edafoclimáticas. El análisis de correlación de las variables edafoclimáticas de la zona cafetera estudiada, permitió encontrar alto grado de asociación entre las variables radiación fotosintéticamente activa (RFA) y precipitación $\left(-0,84^{* *}\right)$, tal como fue demostrado por Urrego y Delvalle, (2001) y Ruiz (2005), quienes manifiestan que existe una relación inversa entre el brillo solar y la precipitación.
Igual situación se observó entre RFA y altitud $(-0,80)$; según Griffin (2001) y Sendiña y Pérez, (2006) existe una relación inversa entre la altitud y el brillo solar recibido por unidad de área, la altitud en las condiciones de estudio está asociada con mayor nubosidad, lo cual explica la asociación negativa con la variable RFA, que también presentó alto grado de asociación con el porcentaje de broca total $\left(0,79^{* *}\right)$.

Este resultado concuerda con Trojer y Gómez (1965) cuando afirman que la temperatura (TMP) y la altitud son factores que influyen mucho en la duración del ciclo biológico de la broca; también fueron importantes los niveles de asociación entre temperatura y altitud $\left(-0,93^{* *}\right)$, igualmente Boshell (1982), Wintgens (2004) y Apráez y Achicanoy (2015), reportan que existe una relación inversa entre la temperatura y la altitud. La TMP y M.O del suelo $\left(-0,90^{* *}\right)$, la altitud y M.O del suelo $\left(0,91^{* *}\right)$ fueron variables con alto grado de asociación, lo cual concuerda con Sánchez et al (2005) quienes afirman que existe una relación directa entre altitud y materia orgánica, asociada a una disminución en la temperatura y a un aumento de la precipitación.

También mostraron alta correlación la altitud y el porcentaje de broca total $\left(-0,86^{* *}\right)$, lo cual concordó con Soto et al., (2002), Díaz et al., (2014) y Bosselmann et al., (2009), cuando afirman que la presencia de la broca del café aumenta al disminuir la altitud debido a que se presenta mayor temperatura.

El grado de asociación de estas variables edafoclimaticas con la variable calidad de taza (puntaje) fue bajo, destacándose la correlación entre el contenido de fosforo y la calidad, con el valor de $-0,50^{* *}$ (Tabla 1 ). 
Tabla 1. Análisis de correlación entre las variables edafoclimáticas, de cultivo y la calidad de taza.

\begin{tabular}{|c|c|c|c|c|c|c|c|c|c|c|c|c|c|c|c|c|c|}
\hline & V1 & V2 & V3 & V4 & V5 & V6 & V7 & V8 & V9 & V10 & V11 & V12 & V13 & V14 & V15 & V16 & V17 \\
\hline V1 & 1,00 & & & & & & & & & & & & & & & & \\
\hline V2 & 0,73 & 1,00 & & & & & & & & & & & & & & & \\
\hline V3 & $-0,84$ & $-0,25$ & 1,00 & & & & & & & & & & & & & & \\
\hline V4 & -080 & $-0,93$ & 0,40 & 1,00 & & & & & & & & & & & & & \\
\hline V5 & $-0,46$ & $-0,07$ & 0,59 & 0,20 & 1,00 & & & & & & & & & & & & \\
\hline V6 & 0,28 & 0,47 & $-0,03$ & $-0,43$ & $-0,54$ & 1,00 & & & & & & & & & & & \\
\hline V7 & $-0,65$ & $-0,90$ & 0,21 & 0,91 & 0,23 & $-0,57$ & 1,00 & & & & & & & & & & \\
\hline V8 & 0,26 & 0,43 & $-0,02$ & $-0,20$ & $-0,24$ & 0,37 & $-0,28$ & 1,00 & & & & & & & & & \\
\hline V9 & 0,38 & 0,29 & $-0,30$ & $-0,36$ & $-0,43$ & 0,66 & $-0,22$ & $-0,07$ & 1,00 & & & & & & & & \\
\hline V10 & 0,66 & 0,61 & $-0,45$ & $-0,70$ & $-0,07$ & 0,23 & $-0,71$ & 0,01 & $-0,01$ & 1,00 & & & & & & & \\
\hline V11 & 0,42 & 0,62 & $-0,10$ & $-0,64$ & $-0,64$ & 0,23 & $-0,72$ & $-0,07$ & $-0,05$ & 0,91 & 1,00 & & & & & & \\
\hline V12 & $-0,09$ & $-0,30$ & $-0,11$ & 0,46 & 0,46 & $-0,25$ & 0,59 & 0,28 & 0,11 & $-0,68$ & $-0,73$ & 1,00 & & & & & \\
\hline V13 & 0,18 & 0,24 & $-0,07$ & $-0,42$ & -042 & $-0,13$ & $-0,28$ & $-0,50$ & 0,11 & 0,39 & 0,48 & $-0,36$ & 1,00 & & & & \\
\hline V14 & 0,06 & 0,24 & 0,11 & $-0,19$ & $-0,19$ & 0,09 & $-0,08$ & 0,48 & $-0,04$ & 0,06 & 0,05 & 0,10 & 0,34 & 1,00 & & & \\
\hline V15 & 0,79 & 0,71 & $-0,57$ & $-0,86$ & $-0,86$ & 0,05 & $-0,74$ & $-0,04$ & 0,08 & 0,78 & 0,66 & $-0,47$ & 0,62 & 0,17 & 1,00 & & \\
\hline V16 & 0,51 & 0,38 & $-0,42$ & $-0,46$ & $-0,46$ & 0,05 & $-0,44$ & $-0,24$ & 0,30 & 0,18 & 0,18 & 0,06 & 0,48 & $-0,22$ & 0,57 & 1,00 & \\
\hline V17 & $-0,40$ & 0,02 & 0,58 & 0,06 & 0,06 & $-0,26$ & $-0,04$ & $-0,23$ & $-0,39$ & 0,00 & 0,25 & $-0,39$ & $-0,16$ & $-0,44$ & -017 & $-0,29$ & 1,00 \\
\hline
\end{tabular}

V1= Radiación fotosintéticamente activa (RFA); V2= Temperatura (TMP); V3= Precipitación (RNF); V4= Altitud; V5= Edad del cultivo (meses); V6= Densidad de siembra (Arboles/Ha); V7= Materia orgánica del suelo; V8= Fosforo en el suelo; V9= Potasio en el suelo; V10= Calcio en el suelo; V11= Magnesio en el suelo; V12=Aluminio en el suelo; V13= Puntaje (\%); V14= Peso de 100 granos c.p.s (g); V15=\%Broca total; V16=\% Pasillas; V17=Relación Fruto/c.p.s (\%)

Esta correlación negativa entre el contenido de fósforo y la calidad de taza es contraria a lo afirmado por Santoyo et al. (1996) cuando dice que las deficiencias de fósforo disminuyen la calidad incrementando el vaneamiento y los granos deformes y Rosas et al. (2008) quienes afirman que el fosforo influye en la calidad sensorial del café, específicamente en su aroma, aspecto de gran relevancia cafetera. Así mismo, estos resultados difieren de Wintgens (2004), quien afirma que la calidad del café está definida por las condiciones de altitud y temperatura. Sin embargo, por la naturaleza multifactorial de la calidad de café, Avelino et al. (2002), Buenaventura y Castaño (2002), Wintgens (2004) y Suarez et al. (2015), consideran que no es posible atribuir a unas pocas variables la calidad física y organoléptica de la bebida.

Según Buenaventura y Castaño (2002), no existe un efecto lineal significativo que describa el comportamiento de las variables organolépticas con respecto a la altitud, demos- trando que no existe una influencia proporcional ni directa sobre las calidades del café.

Los resultados de esta investigación, en cuanto al grado de asociación entre la altitud y la calidad en taza (puntaje), fue bajo con una correlación de $(-0,42)$, esta relación inversamente proporcional difiere con lo descrito por Avelino et al. (2005), en el Valle de Orosí, Costa Rica, quien estableció que los cafés ubicados a bajas alturas (1020 y $1250 \mathrm{msnm}$ ), se caracterizan por tener un sabor más ácido con respecto al café procedente de las regiones de mayor altura (1550 y $1780 \mathrm{msnm}$ ). Igualmente Lara y Vasst (2007) determinaron que la calidad organoléptica (aroma, fragancia, sabor, sabor residual, cuerpo) fue influenciada positivamente en el rango altitudinal de 950 hasta 1255 msnm en la Región Norcentral de Nicaragua. El análisis de componentes principales permitió agrupar el mayor porcentaje de la variabilidad $(71,33 \%)$ en tres grandes factores (Tabla 2). 
Tabla 2. Representación porcentual de la variabilidad observada en las variables cuantitativas relacionadas con el clima, suelo, cultivo y calidad.

\begin{tabular}{ccccl}
\hline Factores & $\begin{array}{c}\text { Valor } \\
\text { propio }\end{array}$ & Porcentaje & $\begin{array}{c}\text { Porcentaje } \\
\text { acumulado }\end{array}$ & \\
\hline 1 & 6,7371 & 39,63 & 39,63 & $* * * * * * * * * * * * * * * * * * * * * * * * * * * * * * * *$ \\
2 & 3,2498 & 19,12 & 58,75 & $* * * * * * * * * * * * * *$ \\
3 & 2,1387 & 12,58 & 71,33 & $* * * * * * * * * * * *$ \\
\hline
\end{tabular}

El factor uno, permitió explicar el 39,63\% de la variabilidad total y estuvo conformado principalmente por las variables RFA $(0,86)$, temperatura $(0,86)$, altitud $(-0,95)$, M.O del suelo $(-0,90)$ y calcio en el suelo $(0,83)$. Estos resultados permiten afirmar que las variables más importantes en la conformación del componente uno, son variables de tipo edafoclimática.

El factor dos, que representó el 19,12\% de la variación se conformó por las variables Edad del cultivo (meses) $(0,77)$ y Aluminio en el suelo $(-0,64)$; el factor tres, que explica el $12,58 \%$ de la variación en la información generada por el estudio se correlacionó principalmente con las variables Fosforo en el suelo $(-0,77)$, Densidad de siembra $(-0,54)$ y puntaje $(0,51)$ (Tabla 3$)$.

El análisis clúster permitió identificar tres grandes grupos de variables con características intragrupales semejantes y diferentes entre los grupos (Figura 1).

En el grupo uno se ubicaron las fincas La playa, Zapatero y Propia, las cuales se destacaron por presentar valores por encima del promedio general en las variables RFA $\left(955,99 \mu \mathrm{mol} / \mathrm{m}^{2} / \mathrm{s}\right)$, broca total $(4,26 \%)$, temperatura $\left(21,34^{\circ} \mathrm{C}\right)$ y Calcio en el suelo $(4,93 \mathrm{cmolc} / \mathrm{Kg})$, con una significancia estadística $\mathrm{p}<0,05$; así mismo los promedios de este grupo presentaron menores valores que los promedios generales en las variables
RNF (616,1 mm), altitud (1472,67 msnm) y M.O del suelo $(3,25 \%)$, con significancia estadística $(\mathrm{p}<0,05)$ (Tabla 4).

Tabla 3. Tabla de correlación entre las variables y cada uno de los factores principales

\begin{tabular}{cccc}
\hline Variables & $\mathbf{1}$ & $\mathbf{2}$ & $\mathbf{3}$ \\
\cline { 1 - 3 } V1 & 0,86 & $-0,34$ & 0,12 \\
V2 & 0,86 & 0,04 & $-0,34$ \\
V3 & $-0,54$ & 0,52 & $-0,44$ \\
V4 & $-0,95$ & $-0,04$ & 0,11 \\
V5 & $-0,31$ & 0,77 & $-0,02$ \\
V6 & 0,45 & $-0,36$ & $-0,54$ \\
V7 & $-0,90$ & $-0,13$ & 0,28 \\
V8 & $0 ., 14$ & $-0,41$ & $-0,77$ \\
V9 & 0,34 & $-0,53$ & 0,05 \\
V10 & 0,83 & 0,32 & $-0,01$ \\
V11 & 0,73 & 0,58 & $-0,10$ \\
V12 & $-0,49$ & $-0,64$ & 0,11 \\
V13 & 0,46 & 0,35 & 0,51 \\
V14 & 0,15 & $-0,14$ & $-0,34$ \\
V15 & 0,90 & 0,15 & 0,27 \\
V16 & 0,52 & $-0,18$ & 0,56 \\
V17 & $-0,21$ & 0,80 & $-0,22$ \\
\hline
\end{tabular}

V1= RFA (Radiación fotosintéticamente activa, V2= TMP (Temperatura), V3= Precipitación (RNF), V4= Altitud, V5= Edad del cultivo, V6= Densidad de siembra, V7 = M.O del suelo, V8= Fosforo en el suelo, V9= Potasio en el suelo, V10 $=$ Calcio en el suelo, V11= Magnesio en el suelo, V12= Aluminio en el suelo, V13= Puntaje de taza) (\%); V14= Peso de 100 granos c.p.s (g); V15=\%Broca total; V16=\% Pasillas; V17=Relación Fruto/c.p.s (\%). 


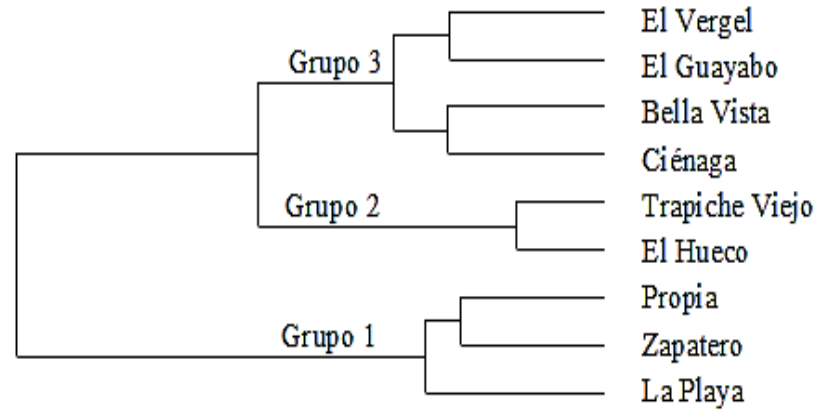

Figura 1. Dendograma clasificatorio de las fincas relacionadas en el presente estudio, con base en las variables estudiadas.

El grupo dos (predios Trapiche Viejo y El Hueco), se caracterizó por presentar mayores valores en la relación fruto:grano $(68,75 \%)$, edad del cultivo (70,50 meses) y RNF $(618,8$ $\mathrm{mm}$ ), comparados con los promedios generales que fueron de $61,67 \%, 64,56$ meses y $586,8 \mathrm{~mm}$, respectivamente.
Finalmente, el tercer grupo, conformado por los predios Ciénaga, Bella vista, El Guayabo y El Vergel presentó mayores valores en altitud (1867,50 msnm) y M.O del suelo (8,93\%), variables cuyos promedios generales fueron de 1676,78 msnm y 6,08\% respectivamente; este mismo grupo, presentó valores inferiores a los promedios generales en las variables TMP $\left(18,92^{\circ} \mathrm{C}\right)$, broca total $(0,48 \%)$, magnesio en el suelo $(0,26 \mathrm{cmolc} / \mathrm{kg})$, calcio en el suelo $(1,50 \mathrm{cmolc} / \mathrm{kg})$ y PAR $\left(722,46 \mu \mathrm{mol} / \mathrm{m}^{2} / \mathrm{s}\right)$, con una significancia de $\mathrm{p}<0,05$ (Tabla 4 ).

\section{Variables relacionadas con el beneficio del café}

El análisis de la tabla de Burt (López y Fachelli, 2013), (Tabla 5), mostró que con una fermentación de 16 - 17 horas, el $25 \%$ de las muestras dieron puntajes entre 61 y 70 , el

Tabla 4. Descripción de las características principales que determinan a cada uno de los grupos establecidos, con base en las variables analizadas.

\begin{tabular}{cccccc}
\hline \multirow{2}{*}{ Probabilidad } & \multicolumn{2}{c}{ Promedio } & \multicolumn{2}{c}{ Desviación } & \multirow{2}{*}{ Variable } \\
& Grupal & General & Grupal & General & \\
\hline GRUP0 1 & & & & & \\
0,002 & 955,99 & 802,37 & 0,00 & 108,75 & V1 \\
0,013 & 4,26 & 2,18 & 1,38 & 1,88 & V15 \\
0,024 & 21,34 & 20,1 & 0,00 & 1,26 & V2 \\
0,034 & 4,93 & 3,11 & 1,39 & 1,99 & V10 \\
0,039 & 3,25 & 6,08 & 0,99 & 3,22 & V7 \\
0,014 & 1472,67 & 1676,78 & 19,60 & 186,06 & V4 \\
0,007 & 616,1 & 586,8 & 0,00 & 0,02 & V3 \\
GRUP0 2 & & & & & \\
0,016 & 68,75 & 61,67 & 0,75 & 4,97 & V17 \\
0,031 & 70,50 & 64,56 & 1,50 & 4,81 & V5 \\
0,032 & 618,8 & 586,8 & 0,00 & 0,02 & V3 \\
GRUP0 3 & & & & & \\
0,005 & 1867,50 & 1676.78 & 82.58 & 186,06 & V4 \\
0,012 & 8,93 & 6.08 & 2.65 & 3,22 & V7 \\
0,032 & 722,46 & 802.37 & 5.37 & 108,75 & V1 \\
0,021 & 1,5 & 3.11 & 0.56 & 1,99 & V10 \\
0,018 & 0,26 & 0.95 & 0.12 & 1,83 & V11 \\
0,011 & 0,48 & 2.18 & 0.58 & 1,88 & V15 \\
0,009 & 18,92 & 20.1 & 0.96 & 1,26 & V2 \\
\hline
\end{tabular}

1= Radiación fotosintéticamente activa (RFA); V2= Temperatura (TMP); V3= Precipitación (RNF); V4= Altitud; V5= Edad del cultivo (meses); V7= Materia orgánica del suelo; V10= Calcio en el suelo; V11= Magnesio en el suelo; V15=\%Broca total; V17=Relación Fruto/c.p.s (\%). 
$75 \%$ restante, puntajes del 71 al 80; cuando se incrementaron las horas de fermentación a 18 - 19 horas, los puntajes se distribuyeron uniformemente en las tres categorías, con un $33,3 \%$ de las muestras en cada una de ellas y cuando la fermentación duró 20 horas, los porcentajes de calidad disminuyeron uniformemente en las categorías de calidad media (61 - 70 puntos), alta (71 - 80 puntos) con $50 \%$ de las muestras en cada una de ellas.

Song-Suk Kim (2014), Peñuela et al, (2010), Rodríguez et al (2014), Duicela et al., (2009), consideran que la fermentación es una de las etapas determinantes sobre la calidad del grano; Córdoba y Guerrero, (2016), Izquierdo (2015) y Beckley, (2010) afirman que cuando la fermentación natural está bien controlada, se obtiene un café de buena calidad en taza, Puerta (2010) considera que fermentaciones prolongadas o incompletas producen defectos en la calidad organoléptica del producto final, cambios indeseables de color (pergamino manchado), densidad (pérdida de peso), olor y acidez, que producen una bebida de mala calidad.

Tabla 5. Correspondencias múltiples (\%) entre algunas variables seleccionadas y la variable puntaje como expresión de la calidad

\begin{tabular}{cccc}
\hline Variables & V20 = 1 & V20 = & V20 = 3 \\
\hline V4 $=1$ & 0,0 & 25,0 & 75,0 \\
V4 = 2 & 33,3 & 33,3 & 33,3 \\
V4 = 3 & 0,0 & 50,0 & 50,0 \\
\hline V6 $=1$ & 0,0 & 50,0 & 50,0 \\
V6 $=2$ & 0,0 & 25,0 & 75,0 \\
V6 $=3$ & 33,3 & 33,3 & 33,3 \\
\hline
\end{tabular}

V4=1=16-17 horas de fermentación; V4=2=18-19 horas de fermentación; V4=3=>20 horas de fermentación; V6=1= 10-19 Horas de secado al sol; V6=2 $=20-25$ Horas de secado al sol; V6 $=3=26-30$ Horas de secado al sol; $V 20=1=$ puntaje entre 50 y $60 ; \mathrm{V} 20=2=$ Puntaje entre 61-70; V20=3= Puntaje entre 71-80.
Al comparar el tiempo de secado al sol con la calidad, expresada como puntaje, se determinó que con 10 - 19 horas de sol, un 50\% las muestras analizadas alcanzaron puntajes medios de 61 - 70 y el otro $50 \%$, puntajes altos (71 - 80); con 20 - 25 horas de secado al sol, el $25 \%$ de las muestras presentó puntajes medios y el $75 \%$ de los puntajes altos; con más horas de sol, los puntajes se distribuyeron equitativamente en las tres categorías.

Esto concuerda con Ferro et al. (1999), cuando afirman que el secado tiene una importancia decisiva en la obtención de un grano comercializable de alta calidad, garantizando su buen sabor y aroma.

El proceso de beneficio del café es crucial para la calidad del producto, en especial sus etapas de fermentación y secado, las cuales tienen relación directa con la calidad física y organoléptica del grano de café (Banegas, 2009).

El análisis de correspondencias múltiples permitió reducir las 25 variables a tres componentes principales que explican un total de $63,41 \%$ de la varianza total. El factor 1 explicó el $27,83 \%$, el factor 2 el $22,48 \%$, el factor 3 el 13,10\% de la variación (Tabla 6).

Según la tabla de contribución de las variables con la formación del factor (Tabla 7) las variables temperatura ambiente $\left({ }^{\circ} \mathrm{C}\right)$, horas de fermentación y horas de secado al sol, fueron las variables que más contribuyeron en la formación del factor 1 , todos con un valor de 7,4 . El factor 2, estuvo conformado principalmente por las variables temperatura ambiente $(10,5)$, humedad relativa $(7,8)$, horas de fermentación $(8,1)$, peso de café lavado $(8,6)$, horas de secado al sol $(10,5)$, porcentaje de merma $(10,4)$, mientras que el factor 3 , está representado en su mayoría por las variables aroma $(9,3)$, cuerpo $(18,7)$ y puntaje $(18,9)$. (Tabla 7). 
Tabla 6. Distribución de la variabilidad en tres factores principales.

\begin{tabular}{ccccl}
\hline Factores & $\begin{array}{c}\text { Valor } \\
\text { propio }\end{array}$ & Porcentaje & $\begin{array}{c}\text { Porcentaje } \\
\text { acumulado }\end{array}$ & \\
\hline 1 & 0,4119 & 27,83 & 27,83 & $* * * * * * * * * * * * * * * * * * * *$ \\
2 & 0,3327 & 22,48 & 50,31 & $* * * * * * * * * * * * * *$ \\
3 & 0,1938 & 13,10 & 63,41 & $* * * * * * * * *$ \\
\hline
\end{tabular}

Tabla 7. Contribuciones acumuladas de las variables categorizadas a la conformación de los tres primeros factores principales.

\begin{tabular}{|c|c|c|c|c|c|c|c|c|c|c|}
\hline Categoría & & & 1 & 2 & 3 & 4 & 5 & 1 & 2 & 3 \\
\hline $\mathrm{V} 2=1$ & 0,89 & 3,50 & 0,38 & $-1,70$ & 0,34 & $-0,19$ & $-0,07$ & 0,3 & 7,8 & 0,5 \\
\hline $\mathrm{V} 2=2$ & 1,78 & 1,25 & $-0,94$ & 0,28 & 0,13 & 0,27 & $-0,25$ & 3,8 & 0,4 & 0,2 \\
\hline \multirow[t]{2}{*}{$V 2=3$} & 1,33 & 2,00 & 1,00 & 0,76 & $-0,40$ & $-0,24$ & 0,39 & 3,3 & 2,3 & 1,1 \\
\hline & & & & & & & & 7,4 & 10,5 & 1,8 \\
\hline V3=1 & 1,78 & 1,25 & 0,44 & 0,76 & $-0,33$ & $-0,22$ & 0,08 & 0,8 & 3,1 & 1,0 \\
\hline V3=2 & 0,89 & 3,50 & $-1,02$ & 0,09 & 0,03 & 1,13 & 0,20 & 2,2 & 0,0 & 0,0 \\
\hline \multirow[t]{2}{*}{ V3=3 } & 1,33 & 2,00 & 0,10 & $-1,08$ & 0,42 & $-0,46$ & $-0,24$ & 0,0 & 4,7 & 1,2 \\
\hline & & & & & & & & 3.1 & 7.8 & 2.2 \\
\hline $\mathrm{V} 4=1$ & 1,78 & 1,25 & $-0,94$ & 0,28 & 0,13 & 0,27 & $-0,25$ & 3,8 & 0,4 & 0,2 \\
\hline $\mathrm{V} 4=2$ & 1,33 & 2,00 & 0,50 & $-1,08$ & $-0,67$ & $-0,20$ & $-0,03$ & 0,8 & 4,7 & 3,1 \\
\hline \multirow[t]{2}{*}{$V 4=3$} & 0,89 & 3,50 & 1,13 & 1,05 & 0,75 & $-0,24$ & 0,55 & 2,8 & 3,0 & 2,6 \\
\hline & & & & & & & & 7,4 & 8,1 & 5,8 \\
\hline $\mathrm{V} 5=1$ & 1,33 & 2,00 & $-0,01$ & $-1,20$ & 0,24 & 0,57 & 0,40 & 0,0 & 5,70 & 0,4 \\
\hline $\mathrm{V} 5=2$ & 1,78 & 1,25 & $-0,66$ & 0,60 & 0,28 & $-0,50$ & $-0,08$ & 1,19 & 1,9 & 0,7 \\
\hline \multirow[t]{2}{*}{$\mathrm{V} 5=3$} & 0,89 & 3,50 & 1,35 & 0,59 & $-0,92$ & 0,16 & 0,44 & 3,9 & 0,9 & 3,8 \\
\hline & & & & & & & & 5,8 & 8,6 & 5,0 \\
\hline$V 6=1$ & 0,89 & 3,50 & 0,38 & $-1,70$ & 0,34 & $-0,19$ & $-0,07$ & 0,3 & 7,8 & 0,5 \\
\hline$V 6=2$ & 1,78 & 1,25 & $-0,94$ & 0,28 & 0,13 & 0,27 & $-0,25$ & 3,8 & 0,4 & 0,2 \\
\hline \multirow[t]{2}{*}{$V 6=3$} & 0,89 & 2,00 & 1,00 & 0,76 & $-0,40$ & $-0,24$ & 0,39 & 3,3 & 2,30 & 1,1 \\
\hline & & & & & & & & 7,4 & 10,5 & 1,8 \\
\hline V8=1 & 2,67 & 0,50 & $-0,45$ & 0,40 & $-0,26$ & $-0,03$ & 0,18 & 1,3 & 1,30 & 0,9 \\
\hline V8=2 & 0,89 & 3,50 & 0,38 & $-1,70$ & 0,34 & $-0,19$ & $-0,07$ & 0,3 & 7,8 & 0,5 \\
\hline \multirow[t]{2}{*}{$V 8=3$} & 0,44 & 8,00 & 1,94 & 1,01 & 0,86 & 0,55 & $-0,94$ & 4,1 & 1,40 & 1,7 \\
\hline & & & & & & & & 5,7 & 10,4 & 3,2 \\
\hline $\mathrm{V} 11=1$ & 2,22 & 0,80 & $-0,04$ & $-0,47$ & 0,38 & $-0,07$ & 0,53 & 0,0 & 1,4 & 1,7 \\
\hline $\mathrm{V} 11=2$ & 1,33 & 2,00 & $-0,59$ & 0,44 & $-0,93$ & $-0,07$ & $-0,58$ & 1,1 & 0,8 & 5,9 \\
\hline \multirow[t]{2}{*}{$V 11=3$} & 0,44 & 8,00 & 1,94 & 1,01 & 0,86 & 0,55 & $-0,94$ & 4,1 & 1,4 & 1,7 \\
\hline & & & & & & & & 5,2 & 3,6 & 9,3 \\
\hline $\mathrm{V} 15=2$ & 3,56 & 0,13 & $-0,09$ & $-0,02$ & 0,34 & 0,03 & $-0,01$ & 0,1 & 0,0 & 2,1 \\
\hline \multirow[t]{2}{*}{$\mathrm{V} 15=3$} & 0,44 & 8,00 & 0,75 & 0,17 & $-2,70$ & $-0,23$ & 0,06 & 0,6 & 0,0 & 16,7 \\
\hline & & & & & & & & 0,7 & 0,0 & 18,7 \\
\hline $\mathrm{V} 20=1$ & 0,44 & 8,00 & 0,75 & 0,17 & $-2,70$ & $-0,23$ & 0,06 & 0,6 & 0,0 & 16,7 \\
\hline $\mathrm{V} 20=2$ & 1,33 & 2,00 & 0,63 & $-0,20$ & 0,44 & 1,16 & 0,00 & 1,3 & 0,2 & 1,3 \\
\hline \multirow{2}{*}{$\mathrm{V} 20=3$} & 2,22 & 0,80 & $-0,53$ & 0,08 & 0,28 & $-0,65$ & $-0,01$ & 1,5 & 0,0 & 0,9 \\
\hline & & & & & & & & 3,4 & 0,2 & 18,9 \\
\hline
\end{tabular}

V2= Temperatura ambiente $\left({ }^{\circ} \mathrm{C}\right), \mathrm{V} 3=$ Humedad relativa $(\%), \mathrm{V} 4=$ Horas de fermentación, V5= Peso de café lavado (\%), V6= Horas de secado al sol, V8= Porcentaje de merma, V11= Aroma, V15= Cuerpo, V20= Puntaje (\%). 
El análisis clúster permitió agrupar las fincas según sus características en tres grandes grupos: el grupo uno estuvo conformado por las fincas La playa, Trapiche viejo, El Hueco, Bella vista; en el grupo dos se ubicaron dos fincas Propia, Zapatero y en el grupo tres, El guayabo, Ciénaga y El vergel (Figura 2).

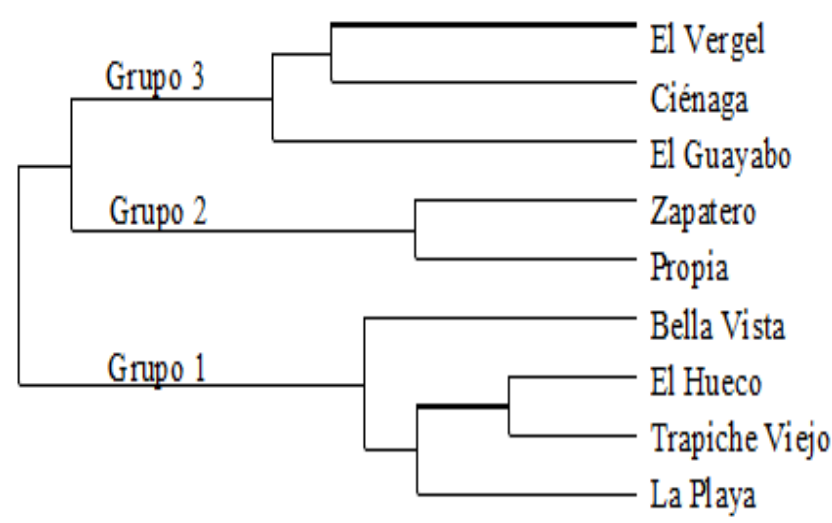

Figura 2. Conformación de grupos de fincas con base en las distancias de Ward para las variables relacionadas con el beneficio del café y calidad de taza.

El grupo uno (Tabla 8) conformado por el $44,4 \%$ de las fincas se caracterizó por realizar la fermentación en 16 - 17 horas, secado al sol durante 20-25 horas y temperaturas durante la fermentación de 25 a $28^{\circ} \mathrm{C}$ y todas con una significancia de $\mathrm{p}<0,05$. El 75\% de estas fincas presentaron altos puntajes de catación en su producto sin significancia estadística $(\mathrm{p}<0,35)$.

En el grupo dos se ubicaron las fincas Propia y Zapatero, que representan el 22,2\% de la población. Estas fincas utilizan 10 - 19 horas de secado al sol, con una temperatura ambiental durante la fermentación de 21 $24^{\circ} \mathrm{C}$ y tiene una merma en el proceso del $18 \%$; éstas variables son características del grupo con una probabilidad de $\mathrm{p}<0,05$ y el grupo tres, conformado por el $33,3 \%$ de la población, presentó un tiempo de secado al sol mayor a 26 horas, un pH de 6 en el mucilago del café al iniciar el proceso de fermentación, temperatura ambiente de 29 $-30^{\circ} \mathrm{C}$ y un 50 a $60 \%$ de humedad relativa; todas estas variables fueron características del grupo tres con una $\mathrm{p}<0,05$ (Tabla 8 y Figura 3).

En la Tabla 8 y Figura 3, puede observarse la ubicación en el plano de factores principales 1 y 2 , de las fincas evaluadas, así como las variables más asociadas con cada finca productora; resalta la ubicación de frutos rojos = 3 (altos puntajes), junto a los predios: Trapiche viejo, El Hueco, La Playa y Bella vista, junto con otras variables asociadas como acidez baja de la bebida, HR de 61 - 65\%, 20 a 25 horas de sol, pH del mucilago al iniciar la fermentación de 4,0, y un porcentaje de merma del $17 \%$ de café almendra con respecto al c.p.s.

Los resultados generales de esta investigación coinciden con Orozco et al. (2011), al no ser posible encontrar que la altitud, está asociada a la calidad de la bebida del café. Existe un conjunto de variables que hacen parte de un sistema muy complejo, por lo cual no es suficiente considerar solo este factor, hay que tomar en cuenta la interacción entre todas las variables y otros que afectan directamente la calidad como las horas de fermentación y el secado. De igual manera, Puerta (2016) confirma que la calidad de la bebida está determinada por las características físicas del grano, la humedad y los defectos; en 216 fincas muestreadas y localizadas en diferentes altitudes (1050 - $2050 \mathrm{msnm}$ ), 15 unidades de suelo, 112 veredas, 35 municipios y siete departamentos, se encontró café de buena y de mala calidad. 
Tabla 8. Descripción de las principales características correspondientes a cada uno de los grupos conformados en el análisis de agrupamiento.

\begin{tabular}{|c|c|c|c|c|}
\hline \multirow{2}{*}{ Probabilidad } & \multicolumn{2}{|c|}{ Porcentajes } & \multirow{2}{*}{ Global } & \multirow{2}{*}{ Variable } \\
\hline & GRP/CAT & CAT/GRP & & \\
\hline GRUPO 1 & 100,00 & 100,00 & 44,44 & $\mathrm{~V} 4=1$ \\
\hline 0,008 & & & & \\
\hline 0,008 & 100,00 & 100,00 & 44,44 & $V 6=2$ \\
\hline 0,008 & 100,00 & 100,00 & 44,44 & $\mathrm{~V} 2=2$ \\
\hline GRUPO 2 & 100,00 & 100,00 & 22,22 & $V 6=1$ \\
\hline 0,028 & & & & \\
\hline 0,028 & 100,00 & 100,00 & 22,22 & $\mathrm{~V} 2=1$ \\
\hline 0,028 & 100,00 & 100,00 & 22,22 & $V 8=2$ \\
\hline GRUPO 3 & 100,00 & 100,00 & 33,33 & $V 6=3$ \\
\hline 0,012 & & & & \\
\hline 0,012 & 100,00 & 100,00 & 33,33 & $\mathrm{~V} 1=3$ \\
\hline 0,012 & 100,00 & 100,00 & 33,33 & $\mathrm{~V} 2=3$ \\
\hline 0,048 & 75,00 & 100,00 & 44,44 & $\mathrm{~V} 3=1$ \\
\hline
\end{tabular}

V1=3: 6 pH del mucilago; V2=1: Temperatura ambiente de $21-24^{\circ} \mathrm{C}$; V2=2: Temperatura ambiente de $25-28^{\circ} \mathrm{C}, \mathrm{V} 2=3$ : Temperatura ambiente de $29-30^{\circ} \mathrm{C}, \mathrm{V} 3=3$ : Humedad relativa de 66-72\%; V4=1: $16-17$ Horas de fermentación,V6=1: 10 - 19 Horas de secado al sol; V6=2: 20-25, V6=3: >26 Horas de secado al sol , V8=2: 18\% de merma.

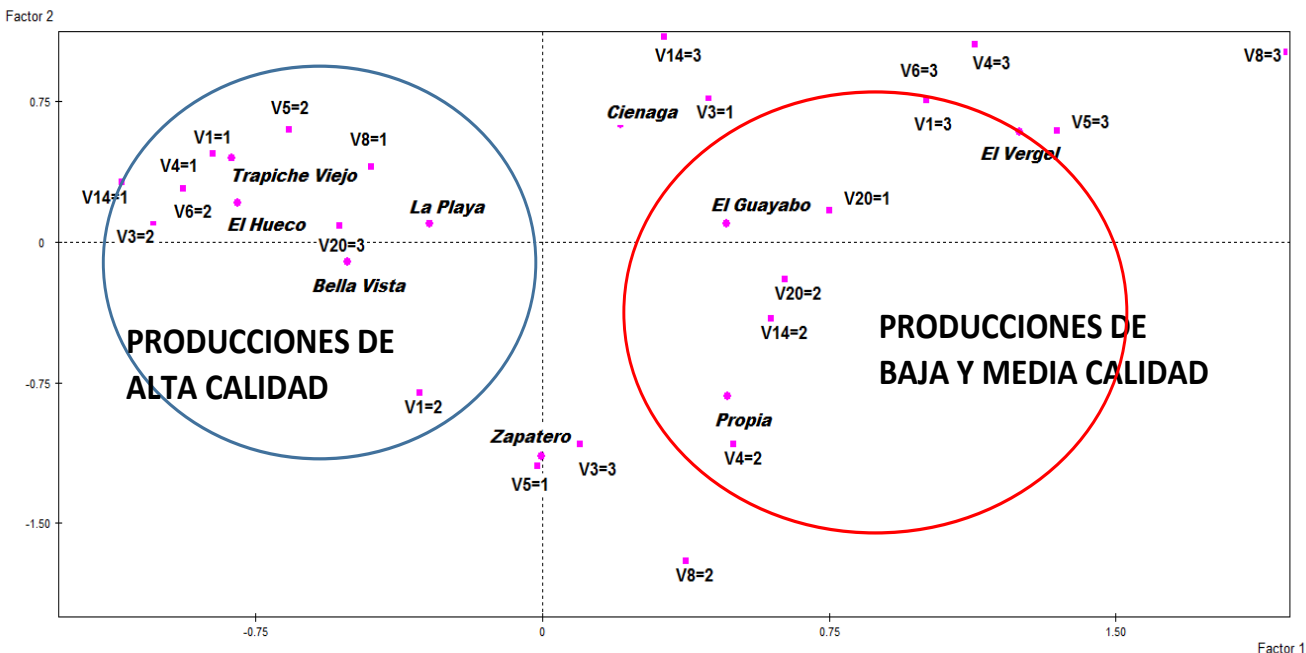

Figura 3. Ubicación de las fincas cafeteras en el plano factorial (factores 1 y 2) con base en las variables de beneficio y de calidad de tasa en el municipio de La Unión, Nariño. 


\section{CONCLUSIONES}

Este estudio permitió determinar que la calidad es una variable multifactorial; la altitud no influyó directamente en la calidad aunque sí se relacionó inversamente con la presencia de broca; menores contenidos de fósforo, menor tiempo de fermentación (16-17 h) y de secado (20-25 h de sol), se asociaron a mayores puntajes de calidad.

\section{AGRADECIMIENTOS}

A los ingenieros Agrónomos Hernando Delgado, Henry Castillo Sánchez y Jaime Noguera Gómez del comité de cafeteros de Nariño.

\section{REFERENCIAS BIBLIOGRÁFICAS}

1. Apráez, G.; Achicanoy, A., 2015. Efecto de las características edafoclimáticas en la calidad nutritiva del pasto brasilero (Phalaris sp.) en el altiplano de Nariño. Revista Ciencia Animal. 9:69 - 82.

2. Aristizabal, A., C.; Duque, O., H. 2006. Determinación de economías de escala en el proceso de beneficio del café en Colombia. Cenicafé. 57(1):17 - 30.

3. Arcila, P.,J.; Farfan, V., F..; Moreno, B., A.M.; Salazar, G., L.F.; Hincapie, G., E. 2007. Sistemas de producción de café en Colombia, Chinchiná. Cenicafé. 55(1):161 - 200.

4. Avelino, J.; Barboza, B.; Araya, J. C.; Fonseca, C.; Davrieux, F.; Guyot, B.; Cilas, C. 2005. Effects of slope exposure, altitude and yield on coffee quality in two altitude terroirs of Costa Rica, Orosí and Santa Maria de Dota. Journal of the Science of Food and Agriculture. 85(11):1869 - 1876.

5. Avelino, J.; Perriot, J.; Guyot, B.; Pineda, C.; Decazy, F.; Cilas, C. 2002: Identifying terroir coffees in Honduras. Research and coffee growing. Montpellier. CIRAD. 7 - 16p.
6. Banegas, K. 2009. Identificación de las fuentes de variación que tienen efecto sobre la calidad del café (Coffea arabica) en los municipios de El Paraiso y Alauca, Honduras. Centro Agronómico Tropical de Investigación y Enseñanza (CATIE), Turrialba, Costa Rica. 58p.

7. Beckley, V. 2010. La fermentación del café. Instituto de defensa del café de Costa Rica, 8:209 - 216.

8. Boshell, J. 1982. Condiciones meteorológicas generales de Colombia. En: Fruticultura tropical. Federecafé. Bogotá. 3 - 8p.

9. Bosselmann, A.; Dons, T.; Oberthur, C.; Olsen, S., Raebild, A.; Usma, H., 2009. The influence of shade trees on coffee quality in small holder coffee agroforestry systems in Southern Colombia. Agriculture, Ecosystems \& Environment. 129(103):253 - 260. doi: https://doi.org/10.15446/acag. v64n4.44641.

10. Buenaventura, S.; Castaño, C., 2002. Influencia de la altitud en la calidad de bebida de muestras de café procedente del ecotopó 206B en Colombia. Revista Cenicafé. 53(2):119 - 131.

11. Comité Departamental de Cafeteros de Nariño. 2008. Informe del Comité Departamental de Cafeteros de Nariño, En: < https://www.federaciondecafeteros.org/ static/files/Caldas4.pdf> 95-102p; consulta: agosto, 2006.

12. Córdoba, N.; Guerrero, J. 2016. Caracterización de los procesos tradicionales de fermentación de café y su efecto sobre la calidad sensorial en el departamento de Nariño. Biotecnología en el sector agropecuario y agroindustrial. 14(2):75 - 83. doi: 10.18684/BSAA.

13. Delgado, P.; Vignoli, J.; Franco, T. 2008 Sediments in coffee extracts. Composition and control by enzymatic hydrolysis. Food Chemistry. 110:168 - 176. doi: 10.1016/j. foodchem.2008.01.029. 
14. Díaz, V.; Perez, J.; Magallanes, R.; Pinson, E.; Coss, M.; Cabrera, M. 2014. Control biológico de la broca del café Hypothenemus hampei Ferrari (Coleóptera: Curculionidae) con diferentes dosis del hongo Beaveria Bassiana (Balls) Vuill. (Moniliales: Moniliaceae) en la Union Juares, Chiapas, México. Vedalia. 15(1):15 - 21.

15. Donnet, M. L.; Weatherspoon, D. D.; Hoehn, J. P. (2007). What adds value in specialty coffee? Managerial implications from hedonic price analysis of Central and South American e-auctions. International Food and Agribusiness Management Review. 10(3):1 - 18.

16. Duicela, G., Corral, C., Farfán, T.; Alcívar, R. 2009. Post cosecha y calidad del café arábigo. Primera edición. ANECAFÉ, USAID. 10p.

17. Ferro, V., Fonseca, S., Abdala, J., Torres, A., Pantoja, J., Bergues, C., 1999. "Análisis de opciones para el secado solar de café. (Parte 1). Aspectos cinéticos. Tecnología Química. 19(3):18 - 25.

18. Griffin, M. 2001. Coffee quality and environmental conditions. Coffee Research Newsletter. 1(3):4 - 6 .

19. Izquierdo, C., 2015. La construcción socio histórica de la calidad del café y del banano de Costa Rica. Un análisis comparado. Revista electro histórica. 16:(1):1890 - 1950.

20. Lara, L., Vaast P., 2007. Effects of altitude, shade, yield and fertilization on coffee quality (Coffea arabica L. var. Caturra) produced in agroforestry systems of the Northern Central Zones of Nicaragua. En: Second International Symposium on Multistrata agroforestry systems with perennial crops: Making ecosystem services count for farmers, consumers and the environment, Costa Rica. IUFRO, CIRAD, CATIE. Turrialba.

21. López-Roldán, P.; Fachelli, S. 2013. Análisis de correspondencias. Metodología de análisis. Papers. Revista de Sociología. 48:9 29.
22. Navia, E., J; Gallego, G., I ; Jojoa, B., I. 2016. Impacto del programa Nespresso AAA en la zona cafetera del municipio de la Unión Nariño. Revista Facultad Ciencias Agropecuarias - FAGROPEC. 8(1):30 - 37.

23. Oberthur, T.; Laderach, P.; Posada, H.; Fisher, M.; Samper, L.; Illera, J. 2011. Regional relationships between inherent coffee quality and growingenvironment for denomination of origin labels in Nariño and Cauca,Colombia, Food Policy. 36:783 - 794. doi: 10.1016/j.foodpol.2011.07.005.

24. Orozco, N., Guacas, A., Bacca, T., 2011. Caracterización de fincas cafeteras por calidad de la bebida y algunas condiciones ambientales y agronómicas. Revista de Ciencias Agrícolas. 28: (2):9 - 17.

25. Peñuela, A., E.; Oliveros, T., C. E.; Sanz, U., J. R., 2010. Remoción del mucílago de café a través de fermentación natural. Cenicafé. 61(2):159 - 173.

26. Puerta, G. 1998. Calidad de las variedades de Coffea arabica L. cultivadas en Colombia. Cenicafé 49(4):265 - 278.

27. Puerta, G. 1999. Influencia del proceso de beneficio en la calidad del café. Revista Cenicafé, 50 (1):78 - 88.

28. Puerta, G. 1996. Escala para la evaluación de la bebida de café verde Coffea arabica $\mathrm{L}$, proceso vía húmeda. Cenicafé. 47(4):231 234.

29. Puerta, G., 2000. Beneficie correctamente su café y conserve la calidad de la bebida. Avances técnicos Cenicafé. 276:1 - 8.

30. Puerta, G., 2010. Rendimientos y calidad de Coffea arabica L., según el desarrollo del fruto y la remoción del mucilago. Cenicafé. 61:67 - 89.

31. Puerta, G. 2016. Calidad física del café de varias regiones de Colombia según altitud, suelos y buenas prácticas de beneficio. Revista Cenicafé 67 (1):7 - 40.

32. Raynolds, L., Murray, D., Heller, A., 2007. Regulating sustainability in the coffee sector: a 
comparative analysis of third-party environmental and social certification initiatives. Agric. Hum. Values. 24:147 - 163. doi: 10.1007/s10460-006-9047-8.

33. Raynolds, L., 2009. Mainstreaming fair trade coffee: from partnership to traceability. World Development. 37(6):1083 - 1093. doi: 10.1016/j.worlddev.2008.10.001.

34. Rebolledo, I.; Vento, L., 2004. Propuesta de agro industrialización del proceso de beneficio del café en el municipio de la Unión (Nariño) de acuerdo a las características de calidad esperadas por el cliente a nivel internacional. Revista Lasallista de Investigación. 12(1):90 - 98.

35. Rodríguez, E., Suarez, J., Duran, E., 2014. Efecto de las condiciones de cultivo, las características químicas del suelo y el manejo de grano en los atributos sensoriales de café (Coffea arabca L.) en taza. Acta agronómica. 64(4):342 - 348. doi: http:// dx.doi.org/10.15446/acag.v64n4.44641.

36. Rosas, A., Escamilla, E.; Ruiz, O., 2008. Relación de los nutrimentos del suelo con las características físicas y sensoriales del café orgánico. Terra Latinoamericana. 26(4):375 - 384 .

37. Ruiz, R., 2005. Desarrollo del racimo y formación de aceite en diferentes épocas del año según las condiciones de la zona norte. Revista Palmas. 26(4):37 - 61.

38. Salazar, E.; Muschler, R.; Sánchez, V.; Jiménez, F. 2000. Calidad de Coffea arabica bajo sombra de Erythrina poepiggiana a diferentes elevaciones en Costa Rica. Agroforestería en las Américas. 7(26):40 - 42.

39. Sánchez, B., Ruiz, M.; Ríos, M. 2005. Materia orgánica y actividad biológica del suelo en relación con la altitud en la cuenca del rio Maracay, estado Aragua. Agronomía tropical. 55(4):507 - 534.

40. Santoyo, C., Díaz, C., Escamilla, P., Robledo, M., 1996. Factores agronómicos y calidad del café. Universidad Autónoma Chapingo. Confederación de productores de café. Chapingo, México. 21p.
41. Sendiña, I.; Pérez, V., 2006. Fundamentos de meteorología. Universidad Santiago de Compostela. España. 194p.

42. Sepúlveda, W., Maza, M.; Mantecón, A., 2013. Information demanded by consumers: New issues in voluntary beef labeling. Acta Alimentaria. 42(2):3 - 10. doi: 10.1556/AAlim.42.2013.2.2.

43. Song-Suk Kim, A. 2014. Comparison of Antioxidant Effects among Non-fermented and Fermented Columbian Coffee, and Luwak Coffee Beans. Korean journal of food and cookery science. 30(6):757 - 766. doi: $10.9724 / \mathrm{kfcs} .2014 .30 .6 .757$.

44. Soto, L., Perfecto, I.; Caballero, J., 2002. Shade over coffee: its effects on berry borer, lveaf rust and spontaneous herbs in Chiapas, Mexico. Agroforestry Systems 55:37 45. doi: 10.1023/A:1020266709570.

45. Suarez, J; Rodríguez, E; Humprey, E. 2015. Efecto de las condiciones de cultivo, las características químicas del suelo y el manejo de grano en los atributos sensoriales de café (Coffea arabica L.) en taza. Acta agronómica. 64(4):329 - 335. doi: https://doi. org/10.15446/acag.v64n4.44641.

46. Trojer, H.; Gómez, L., 1965. "Zonas cafetaleras colombianas susceptibles por sus condiciones climáticas a un ataque de la broca del café", Cenicafé, Centro Nacional de Investigaciones de Café, Chinchiná, Colombia. 16:12 - 30.

47. Urrego, L.; Del Valle, J. 2001. Relación fenología-clima de algunas especies de los humedales forestales (guandales) del pacifico sur colombiano. Interciencia. 26(4):150 - 156.

48. Wintgens, J. 2004. Factors influencing the quality of green coffee. pp.798 - 809. En: J, Wintgens (ed.). Coffee: growing, processing, sustainable production. Alemania, WileyVCH. 